\title{
Phosphorylation of signal transducer and activator of transcription 3 induced by hyperglycemia is different with that induced by lipopolysaccharide or erythropoietin via receptor-coupled signaling in cardiac cells
}

\author{
YU-HSIN CHIU ${ }^{1}$, PO-MING KU ${ }^{2}$, YUNG-ZE CHENG ${ }^{3,4}$, YINGXIAO LI $^{4}$, JUEI-TANG CHENG $^{4,5}$ and HO-SHAN NIU ${ }^{6}$ \\ ${ }^{1}$ Division of Infectious Diseases; ${ }^{2}$ Cardiovascular Center, Department of Internal Medicine, Chi-Mei Medical Center-Liouying, \\ Tainan 73601; Departments of ${ }^{3}$ Emergency Medicine and ${ }^{4}$ Medical Research, Chi-Mei Medical Center, Tainan 71003; \\ ${ }^{5}$ Institute of Medical Sciences, Chang Jung Christian University, Tainan 71101; ${ }^{6}$ Department of Nursing, \\ Tzu Chi University of Science and Technology, Hualien 97005, Taiwan, R.O.C.
}

Received August 17, 2017; Accepted October 25, 2017

DOI: $10.3892 / \mathrm{mmr} .2017 .7973$

\begin{abstract}
The signal transducer and activator of transcription 3 (STAT3) is known to be involved in hypertrophy and fibrosis in cardiac dysfunction. The activation of STAT3 via the phosphorylation of STAT3 is required for the production of functional activity. It has been established that lipopolysaccharide (LPS)-induced phosphorylation of STAT3 in cardiomyocytes primarily occurs through a direct receptor-mediated action. This effect is demonstrated to be produced rapidly. STAT3 in cardiac fibrosis of diabetes is induced by high glucose through promotion of the STAT3-associated signaling pathway. However, the time schedule for STAT3 activation between LPS and high glucose appears to be different. Therefore, the difference in STAT3 activation between LPS and hyperglycemia in cardiomyocytes requires elucidation. The present study investigated the phosphorylation of STAT3 induced by LPS and hyperglycemia in the rat cardiac cell line $\mathrm{H} 9 \mathrm{c} 2$. Additionally, phosphorylation of STAT3 induced by erythropoietin (EPO) via receptor activation was compared. Then, the downstream signals for fibrosis, including the connective tissue growth factor (CTGF) and matrix metalloproteinase (MMP)-9, were determined using western blotting, while the mRNA levels were quantified.
\end{abstract}

Correspondence to: Professor Juei-Tang Cheng, Institute of Medical Sciences, Chang Jung Christian University, Sec. 1, 396 Changrong Road, Gueiren, Tainan 71101, Taiwan, R.O.C.

E-mail: jtcheng@mail.cjcu.edu.tw

Professor Ho-Shan Niu, Department of Nursing, Tzu Chi University of Science and Technology, Sec. 2, 880 Chien-kuo Road, Hualien 97005, Taiwan, R.O.C.

E-mail: nhs580113@yahoo.com.tw

Key words: phosphorylation of signal, lipopolysaccharide, erythropoietin, hyperglycemia, H9c2 cells
LPS induced a rapid elevation of STAT3 phosphorylation in H9c2 cells within $30 \mathrm{~min}$, similar to that produced by EPO. However, LPS or EPO failed to modify the mRNA level of STAT3, and/or the downstream signals for fibrosis. High glucose increased STAT3 phosphorylation to be stable after a long period of incubation. Glucose incubation for $24 \mathrm{~h}$ may augment the STAT3 expression in a dose-dependent manner. Consequently, fibrosis-associated signals, including CTGF and MMP-9 protein, were raised in parallel. In the presence of tiron, an antioxidant, these changes by hyperglycemia were markedly reduced, demonstrating the mediation of oxidative stress. Therefore, LPS- or EPO-induced STAT3 phosphorylation is different compared with that caused by high glucose in H9c2 cells. Sustained activation of STAT3 by hyperglycemia may promote the expression of fibrosis-associated signals, including CTGF and MMP-9, in H9c2 cells. Therefore, regarding the cardiac dysfunctions associated with diabetes and/or hyperglycemia, the identification of nuclear STAT3 may be more reliable compared with the assay of phosphorylated STAT3 in cardiac cells.

\section{Introduction}

The signal transducer and activator of transcription 3 (STAT3) has been demonstrated to be one of the regulators in cardiac dysfunction (1). STAT3 possesses multiple functions, with its central role described as a transcription factor. Moreover, STAT3 has been demonstrated to function as a signaling molecule, as a factor involved in cellular respiration, and as a protein interacting with the mitochondrial pore (2-5). Therefore, in cardiomyocytes, STAT3 plays an important role in survival, growth, sarcomere architecture, energetics, and metabolism (6-8).

Hyperglycemia is important in the pathogenesis of diabetic disorders. Hyperglycemia was found to increase the STAT3 either through the gene expression or the phosphorylation (9). STAT3 is known as a cytoplasmic transcription factor that transmits extracellular signals to the nucleus (10). Activated 
STAT3 in the nucleus binds to specific DNA promoter sequences to regulate the gene expression (11). Recent studies have indicated that hyperglycemia increases STAT3 activation, thereby contributing to the pathophysiology of tissue injury (12). STAT3 activation, increased phosphorylated STAT3 (p-STAT3) and p-STAT3 nuclear translocation, are reportedly some of the underlining mechanisms of STAT3 under high glucose condition. However, p-STAT3 was induced at Y705 and S727 in cells for STAT3 activation by high glucose levels (13). STAT3 has been demonstrated to shuttle between the cytoplasm and nucleus independently of tyrosine phosphorylation (14) while unphosphorylated STAT3 in nucleus also can drive gene expression (15).

Lipopolysaccharide (LPS) is mainly obtained from the outer membrane of gram-negative bacteria, and the inflammatory cytokines produced as a consequence of LPS exposure are implicated in cardiac dysfunction $(16,17)$. The rapid activation of STAT3 by LPS through phosphorylation in cardiomyocytes has been identified (18), and it is suggested as a direct receptor-mediated activation (19). However, STAT3 activation by LPS in hepatocytes is slower than in cardiomyocytes (20). Toll-like receptor 4 (TLR4) is known as the binding site of LPS (21). Activation of TLR4 by LPS has also been indicated to induce an inflammatory response that decreases cardiomyocytes contractility (22). Moreover, the Janus-activated kinase 2 (JAK2) and the STAT3 pathway (JAK2/STAT3 pathway) is also coupled to the signaling of cytokine receptors including TLR4 (23). Otherwise, erythropoietin (EPO) is also produced effectiveness through activation of the specific cell-surface receptor, erythropoietin receptor (EPOR) (24). It has been established that JAK2/STAT3 signaling pathway is also coupled to EPOR (25). Interestingly, agent improves left ventricular performance via activation of JAK2/STAT3 pathway in rats (26). Therefore, we included the effects of EPO in this study, because EPO produced actions also through an activation of receptors, EPOR, which is similar to the action of LPS (27).

Additionally, STAT3 is introduced to involve in cardiac fibrosis of diabetes (28), while high glucose increased STAT3 activated by angiotensin IIhas been demonstrated to be produced mainly through a reactive oxygen species (ROS)-dependent mechanism (29). Recently, the ROS-activated STAT3 pathway has been characterized in early reperfusion of heart (30). High glucose is known as the main factor for inducing diabetes-associated cardiovascular dysfunctions. It seems that activation of STAT3 through phosphorylation by hyperglycemia differs from the promotion of STAT3 via receptor-coupled signaling in the regulation of cardiac function. However, variations in the phosphorylation of STAT3 between high glucose-induced change and promotion by receptor-coupled signaling remained unclear in cardiomyocytes.

In the present study, we focused on STAT3 phosphorylation that is important in regulation of cardiac function. Also, we are interested to know the difference whether STAT3 phosphorylation induced by receptor signaling is varied with that induced by pathologic disorders such as hyperglycemia. Therefore, we used the embryonic rat cardiomyoblast cell line H9c2 which offers the advantage of being an animal-free alternative (31). Moreover, H9c2 expressed TLR4 (32) and EPOR (33). Therefore, it is suitable to apply in the present study.
Additionally, we link it to signals-associated fibrosis, including connective tissue growth factor (CTGF) and metalloproteinase (MMP)-9, to determine its association in cardiac disorders.

\section{Materials and methods}

Cell cultures. It has been confirmed that $\mathrm{H} 9 \mathrm{c} 2$ cells possess the advantage of being an animal-free alternative (31). The H9C2 cells (BCRC, no. 60096) were cultured according to a previous method (34). In brief, H9c2 cells were maintained in Dulbecco's modified Eagle's medium (pH 7.2) supplemented with $10 \%$ fetal bovine serum. The H9c2 cells were plated at a density of $6,000 \mathrm{cells} / \mathrm{cm}^{2}$ and allowed to proliferate in growth medium. The medium was changed every $48 \mathrm{~h}$.

Drug treatment. The cultured $\mathrm{H} 9 \mathrm{c} 2$ cells were treated at indicated times with Salmonella typhosa LPS (Sigma-Aldrich; Merck KGaA, Darmstadt, Germany), as described previously (18). The stock solution of EPO containing epoetin beta (Recormon, 5,000 IU/0.3 ml), purchased from Roche Diagnostics (Mannheim, Germany), was diluted in culture medium. A fresh solution diluted to the indicated dose was applied to treat the H9c2 cells. Incubation of hyperglycemia with $\mathrm{H} 9 \mathrm{c} 2$ cells was also performed according to our previous report (35).

Western blot analysis. Protein was extracted and separated by SDS-PAGE, following our previous method (27). Proteins were detected using antibodies $(1: 1,000)$ against p-STAT3, STAT3, CTGF and MMP-9, while antibody against $\beta$-actin serving as the internal control. After comparing with the marker, the immunoblots of TLR4 (95 kDa), EPOR (55 kDa), p-JAK2 (130 kDa), JAK2 (130 kDa), p-STAT3 (88 kDa), STAT3 (88 kDa), CTGF (38 kDa), MMP-9 (92 kDa) and $\beta$-actin (43 kDa) were then quantified.

Quantitative reverse transcription-polymerase chain reaction ( $q R T-P C R$ ). Total RNA was extracted from cell lysates with TRIzol (Qiagen, Hilden, Germany). Two microgram of total RNA was used for the reverse transcription reaction, along with Superscriptase II (Invitrogen; Thermo Fisher Scientific, Inc., Waltham, MA, USA), oligo-dT, and random primers. Web-based assay-design software from the Universal Probe Library Assay Design Center (http://www. roche-applied-science.com/sis/rtpcr/upl/adc.jsp) was utilized to design TaqMan primer pairs and to select appropriate hybridization probes (Table I). For quantification, real-time PCR analysis was performed using Light Cycler 480 SYBR-Green I Master on a Light Cycler 480 II (Roche Diagnostics). The relative fold changes were quantified using the comparative threshold cycle method, and $\beta$-actin was used as a control, according to our previous reports $(36,37)$.

Statistical analysis. Data were indicated as the mean \pm standard error of the mean (SEM) from the sample number (n) of each group. The differences between two groups were analyzed using a Student's two-sided t-test. A value of $\mathrm{P}<0.05$ was considered to indicate a statistically significant difference. 


\section{Results}

Effects of LPS on the phosphorylation of STAT3 in H9c2 cells. Incubation of LPS dose-dependently induced a marked elevation of STAT3 phosphorylation within $30 \mathrm{~min}$ in $\mathrm{H} 9 \mathrm{c} 2$ cells, as shown in Fig. 1A. The results were obtained from TLR4 through phosphorylated JAK2 (p-JAK2) to STAT3 phosphorylation (Fig. 1A and B). However, the expression of STAT3 was still not modified in terms of both protein and mRNA levels (Fig. 1C). Consequently, the downstream signals both CTGF and MMP-9 were also not activated by LPS in this condition (Fig. 1A and B).

Effects of EPO on the phosphorylation of STAT3 in H9c2 cells. The same incubation of EPO with H9c2 cells also produced a similar change in STAT3 phosphorylation, as shown in Fig. 2A, except that the EPOR was activated by EPO. Additionally, the expression of STAT3 was also not changed by EPO in terms of both protein and mRNA levels (Fig. 2B and C). Similarly, the consequent downstream signals, both CTGF and MMP-9, were also not activated by EPO in this condition.

Effect of hyperglycemia on the phosphorylation of STAT3 in $H 9 c 2$ cells. Incubation of high glucose $(30 \mathrm{mM})$ with $\mathrm{H} 9 \mathrm{c} 2$ cells at the time same as LPS failed to induce changes in STAT3 phosphorylation. Therefore, we incubated H9c2 cells for a longer time with high glucose in the medium. As shown in Fig. 3A, changes in STAT3 phosphorylation were not stable except at $24 \mathrm{~h}$ post-incubation. Both protein and mRNA levels of STAT3 were also markedly elevated after 24-h incubation with high glucose (Fig. 3B).

Additionally, increase of STAT3 expression, both in terms of protein (Fig. 4A) and mRNA (Fig. 4B) levels, was produced in a dose-dependent manner by hyperglycemia after 24-h incubation. However, changes were not observed in H9C2 cells that received similar incubation with manitol $(30 \mathrm{mM})$, which produced the same osmolarity as high glucose $(30 \mathrm{mM})$, as described in our previous report (35). Thus, the possible influence of osmolarity in the changes of STAT3 expression can be excluded.

Moreover, the downstream signals for fibrosis, including CTGF and MMP-9, were also enhanced by hyperglycemia in the same dose-dependent fashion (Fig. 4A). This change was also not related to osmolarity as shown in manitol-treated cells. However, fibrosis-related signals were not modified in $\mathrm{H} 9 \mathrm{c} 2$ cells treated with LPS or EPO at the effective dose in above.

Mediation of oxidative stress in hyperglycemia increased expressions of STAT3 in H9c2 cells. We applied the antioxidant, tiron, to examine the role of oxidative stress in the changes of STAT3 expression by hyperglycemia. As shown in Fig. 5A, tiron inhibits the elevation of STAT3 activation in a dose-related manner. Moreover, the promotion in mRNA level of STAT3 by hyperglycemia was also reduced by tiron in the same fashion (Fig. 5B). Consequently, fibrosis-associated signals, including CTGF and MMP-9, elevated by hyperglycemia were markedly reduced by tiron in the same manner (Fig. 4A). Mediation of oxidative stress can thus be confirmed.
Table I. Primers used for targets amplification in this study.

\begin{tabular}{lcc}
\hline Target & Primer & Sequence (5'-3') \\
\hline STAT3 & F & 5'-GGCTTCAGCCCCAGAGAC-3' \\
& R & 5'-CTCCAGGTAGCGCGTGTC-3' \\
CTGF & F & 5'-ATGCTGTGAGGAGTGGGTGT-3' \\
& R & 5'-GGCCAAATGTGTCTTCCAGT-3' \\
MMP-9 & F & 5'-TCGTGGCTCTAAACCTGACC-3' \\
& R & 5'-GAGCTGTCGGCTGTGGTT-3' \\
$\beta$-actin & F & 5'-CTCTCTTCCAGCCTTCCTTC-3' \\
& R & 5'-GGTCTTTACGGATGTCAACG-3' \\
\hline
\end{tabular}

F, forward; R, reverse; STAT3, signal transducer and activator of transcription 3; CTGF, connective tissue growth factor; MMP-9, matrix metalloproteinase-9.

\section{Discussion}

In the present study, we demonstrated that STAT3 phosphorylation occurred within $30 \mathrm{~min}$ after exposure to LPS in $\mathrm{H} 9 \mathrm{c} 2$ cells. Additionally, the dose-dependent effect of LPS was produced through TLR4 to link the p-JAK2 for STAT3 phosphorylation; it is fully consistent with the previous reports $(22,26)$. However, LPS did not influence the expressions of STAT3 and the downstream signals, including CTGF and MMP-9. Similar results were also obtained in EPO-treated H9c2 cells except that EPOR was involved in the STAT3 phosphorylation by EPO (24). Otherwise, high glucose increased STAT3 phosphorylation after a longer time, particularly $24 \mathrm{~h}$ post-incubation. Additionally, expression of STAT3 was also augmented by high glucose in a dose-dependent manner after 24-h incubation. In parallel, fibrosis-related signals, including CTGF and MMP-9, were both elevated. Therefore, STAT3 phosphorylation induced by LPS or EPO is quite different from that by hyperglycemia. The possible reason might be due to the treatment of $\mathrm{H} 9 \mathrm{c} 2$ with LPS or EPO may stimulatee cardiac mitochondrial function through a highly regulated, receptor-mediated, eNOS/Akt1 and JAK-STAT-dependent cascade that activates the transcriptional program of mitochondrial biogenesis in a short time (38-40). However, hyperglycemia-induced oxidative stress attenuated the mitochondrial function and affected the proliferation and survival of cells. Increased ROS in diabetes is implicated in the development of diabetic cardiomyopathy (41). To the best of our knowledge, the present study is the first to conduct this finding.

Inflammatory cytokines produced as a consequence of LPS exposure are implicated in myocardial dysfunction $(17,42)$. Paradoxically, sub-lethal doses of LPS provided cardioprotective effects against ischemia-reperfusion injury (43). In addition, STAT3 is demonstrated to be a key modulator of an integrated signaling network in the heart (1). Increase of STAT3 phosphorylation by LPS in a CD14-independent manner has been indicated in cardiomyocytes (18). Moreover, EPO is known to protect heart from chemical damage (44) and to improve experimental heart failure (45). In the present study, we observed that STAT3 phosphorylation is raised by EPO in a way that is similar to LPS in H9c2 cells. Interestingly, 

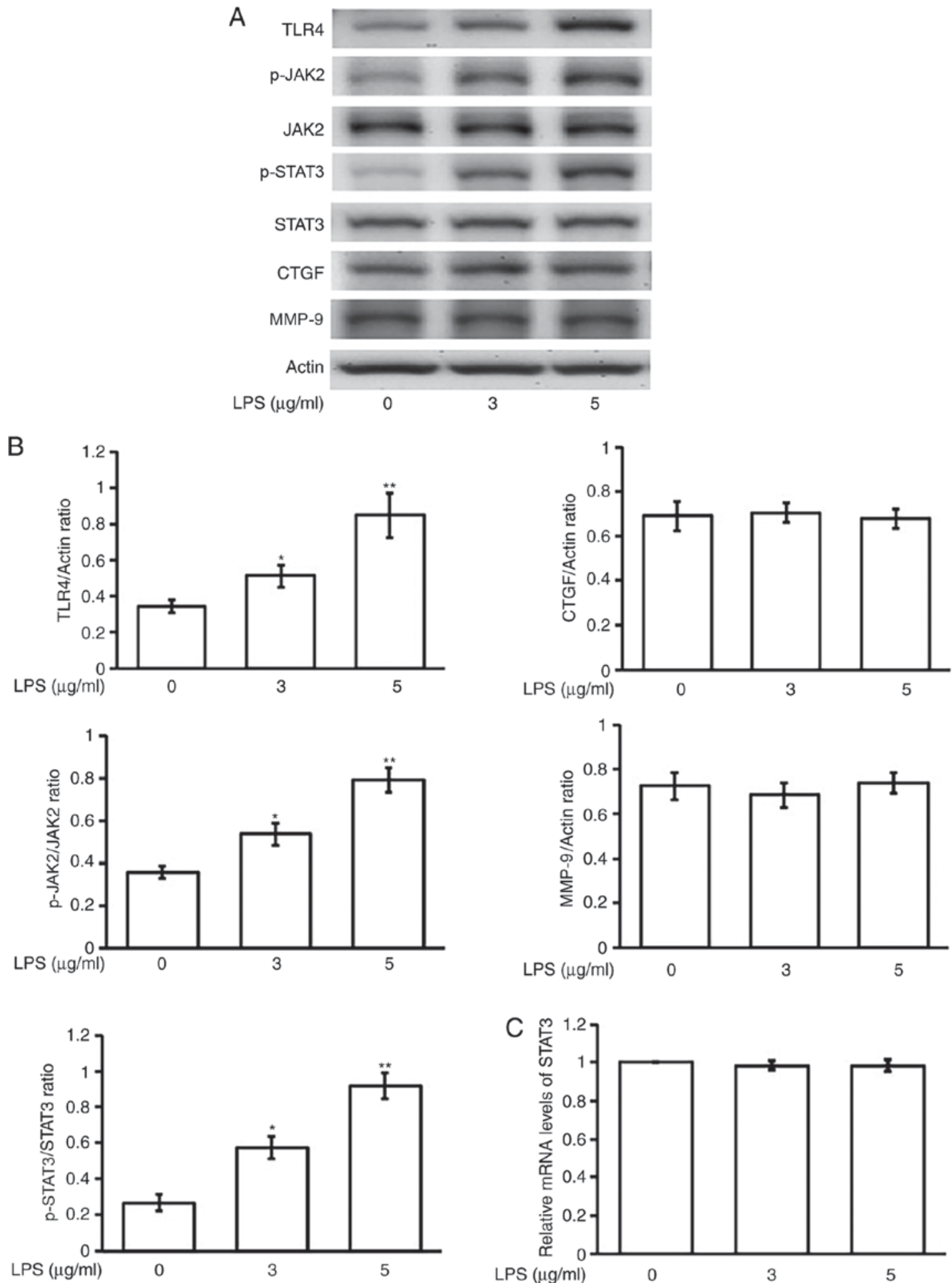

Figure 1. Effects of LPS on the changes in expression of STAT3 and associated signals in H9c2 cells. (A) The representative changes in signals in addition to the p-STAT3, STAT3, CTGF and MMP-9 expressions by LPS at indicated concentration in western blots. (B) The protein levels, using phosphorylated signal over the original one or each signal over $\beta$-actin (Actin), are indicated as mean $\pm \mathrm{SEM}\left(\mathrm{n}=6\right.$ per group) in each column. ${ }^{*} \mathrm{P}<0.05$ and ${ }^{* *} \mathrm{P}<0.01$ compared to the vehicle-treated control shown at 0 concentration. (C) Related mRNA expression as detected using RT-PCR and the quantified mRNA STAT3 level is represented as mean \pm SEM ( $n=6$ per group). LPS, lipopolysaccharide; STAT3, signal transducer and activator of transcription 3; JAK2, Janus-activated kinase 2; p-, phosphorylated; CTGF, connective tissue growth factor; MMP-9, matrix metalloproteinase-9; SEM, standard error of the mean.

both effects were induced by activation of each specific receptors, LPS via TLR4 (21) and EPO through specific receptor EPOR (25). Moreover, both effects on STAT3 phosphorylation were produced through p-JAK2, as described previously (23). Therefore, a rapid increase of p-STAT3 seems helpful in protection against cardiac damage. This view is consistent to previous reports that demonstrated the cardio-protective effects of propofol (46) and morphine (47) through an increase in STAT3 phosphorylation.

In contrast, as shown in Fig. 3, STAT3 phosphorylation was not immediately increased by high glucose, but rather changed in unstable way. After a longer time of incubation with 

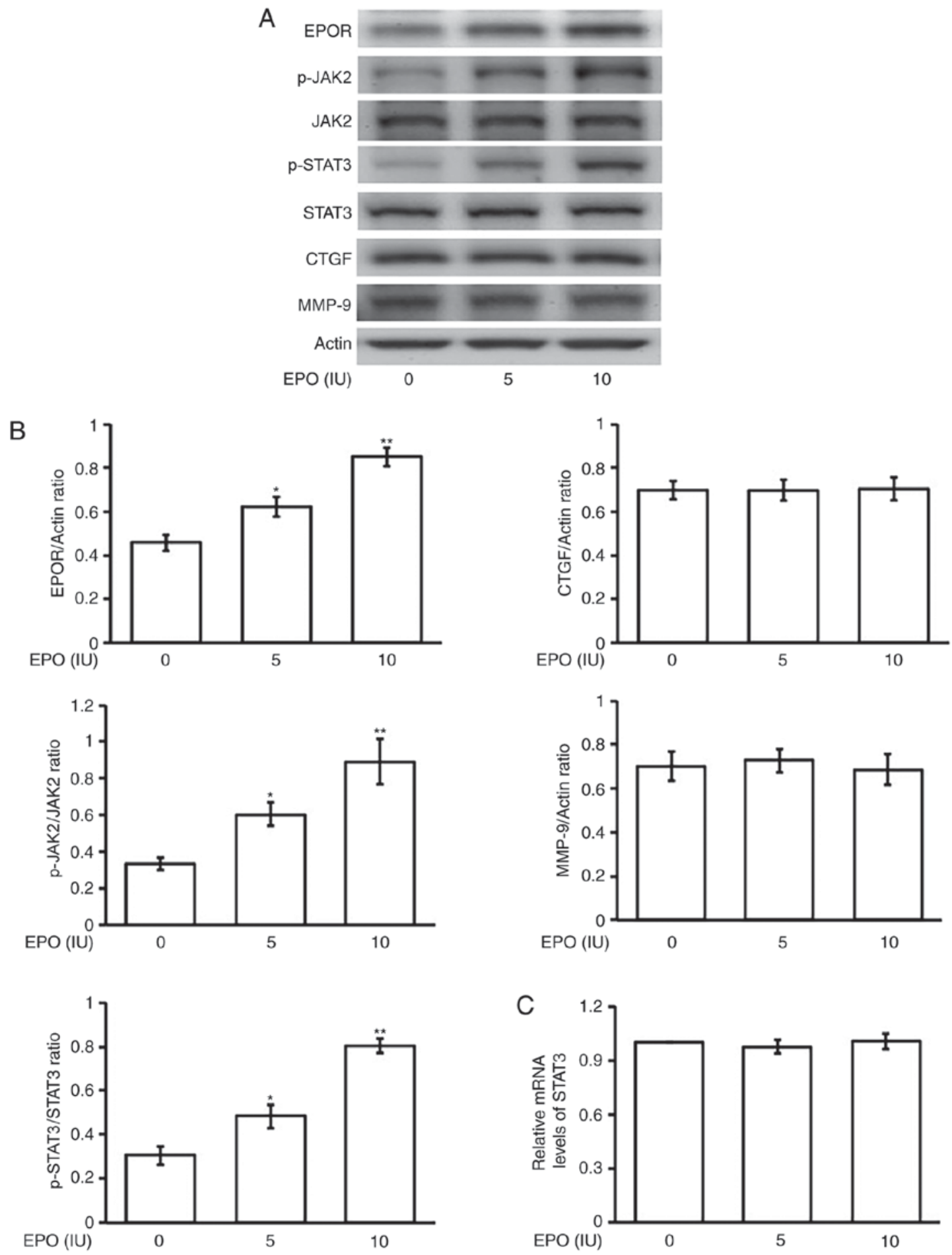

Figure 2. Effects of EPO on the changes in expression of STAT3 and associated signals in H9c2 cells. (A) The representative changes in receptor signals in addition to the p-STAT3, STAT3, CTGF and MMP-9 expressions by EPO at indicated concentration in western blots. (B) The protein levels, using phosphorylated signal over the original one or each signal over $\beta$-actin (Actin), are indicated as mean \pm SEM ( $\mathrm{n}=6$ per group) in each column. ${ }^{*} \mathrm{P}<0.05$ and ${ }^{* *} \mathrm{P}<0.01$ compared to the vehicle-treated control shown at 0 concentration. (C) Related mRNA expression as detected using RT-PCR and the quantified mRNA STAT3 level is represented as mean $\pm \mathrm{SEM}(\mathrm{n}=6$ per group). EPO. erythropoietin; STAT3, signal transducer and activator of transcription 3; p-, phosphorylated; CTGF, connective tissue growth factor; MMP-9, matrix metalloproteinase-9; SEM, standard error of the mean; EPOR; erythropoietin receptor; JAK2, Janus-activated kinase 2 .

H9c2 cells, approximately $24 \mathrm{~h}$ later, STAT3 phosphorylation was stable and markedly increased in high glucose medium. Moreover, expression of STAT3 was also promoted after the same incubation. Basically, phosphorylation is known as the major way for the activation of STAT. Moreover, p-STAT3 has been shown to enter the nucleus easily (48). However, nuclear accumulation of STAT3 without phosporylation has also been demonstrated (49), and the unphosphorylated STAT3 in nucleus may activate gene expression both in cancer and in responses to cytokines (15). Therefore, STAT3 is effective to promote transcription in cardiac cells that have been characterized in this study. We demonstrated that fibrosis-related 


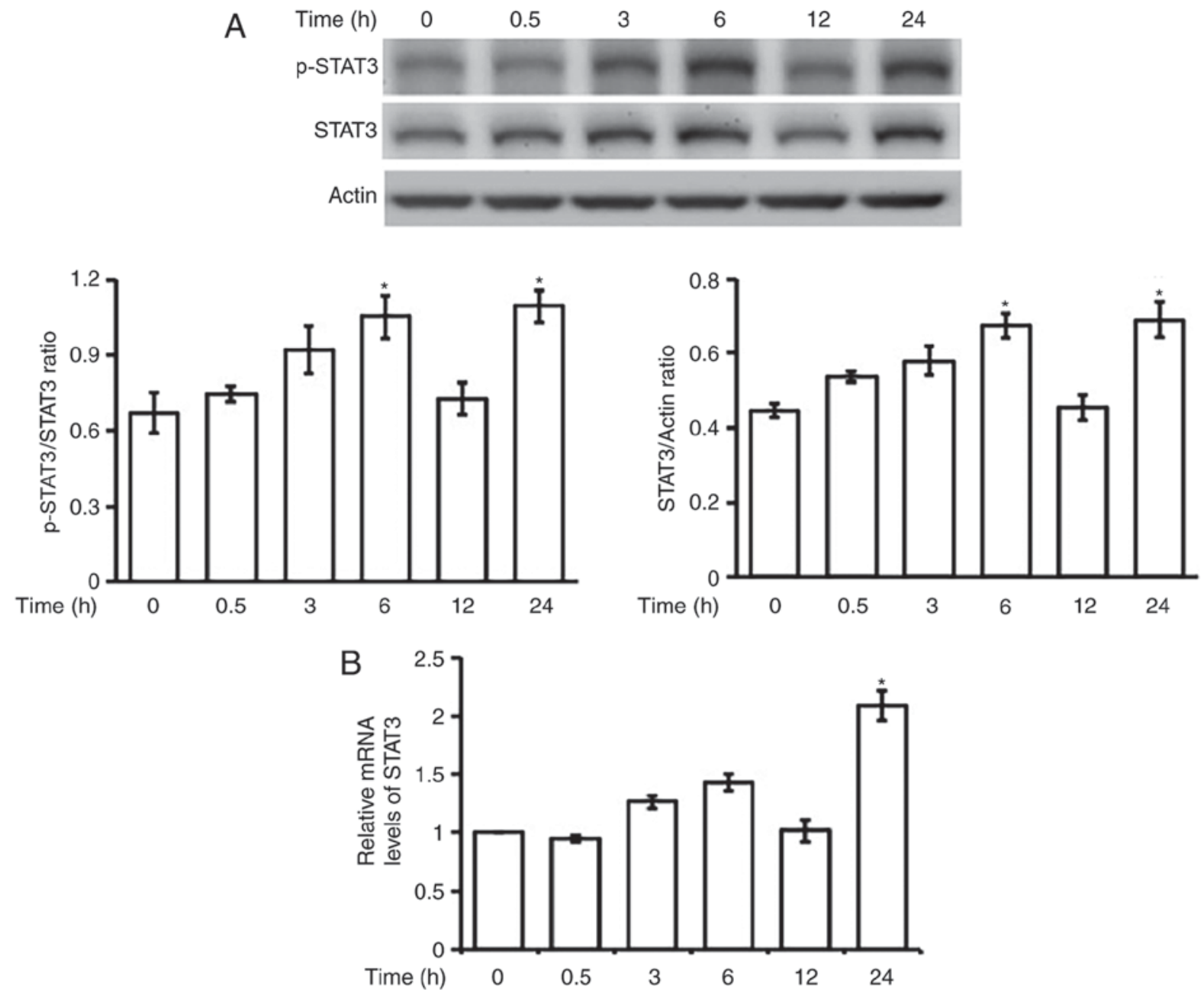

Figure 3. Changes of p-STAT3 and STAT3 expressions in H9c2 cells after exposure to high glucose at indicated time points. (A) The representative changes in p-STAT3 and STAT3 expressions from H9c2 cells after incubation with high glucose $(30 \mathrm{mM})$ at the indicated times $(\mathrm{h}=0-24)$. The protein levels, using p-STAT3 over STAT3 or STAT3 over $\beta$-actin, are indicated as mean \pm SEM ( $n=4$ per group) in each column. (B) Related mRNA expression as detected using RT-PCR and the quantified mRNA level of STAT3 is represented as mean \pm SEM (n=6 per group). ${ }^{*}<<0.05$ compared to the control (at $0 \mathrm{~h}$ ). p-, phosphorylated; STAT3, signal transducer and activator of transcription 3; SEM, standard error of the mean.

signals, including CTGF and MMP-9, were both enhanced in high glucose medium with the increased STAT3. This novel view is useful to explain the role of cardiac fibrosis in diabetes $(50,51)$.

Cardiac fibrosis is a prominent component of diabetic cardiomyopathy $(52,53)$. High glucose has been demonstrated to promote fibrosis in vitro, including changes in MMP activity (28). Hyperglycemia may sustain the progression of heart failure through excessive interstitial myocardial collagen accumulation, thus leading to impaired diastolic and systolic function (54). STAT3 is also shown to mediate the proliferation of cardiac fibroblasts and collagen synthesis induced by high glucose (55). Although STAT3 may participate in the transcription of target genes in ischemia/reperfusion injury (54) and pressure overload hypertrophy (55), the role of STAT3 in cardiac fibrosis induced by hyperglycemia is critical.

The pathogenesis of cardiovascular diseases almost invariably involves, the occurrence of oxidative stress (56), a major cause of progressive cellular sufferance and death. Moreover, diabetic cardiomyopathy is a condition in which oxidative stress seems to play a major pathogenic role (57). Therefore, we focused on the role of oxidative stress in the changes of STAT3 induced by hyperglycemia in H9c2 cells. In the presence of the antioxidant, tiron (58), increase of STAT3 by high glucose was markedly reduced in H9c2 cells. Additionally, the augmented fibrosis-related signals, including CTGF and MMP-9, were also attenuated in parallel. Different to the effects induced by stimulation of receptors, such as LPS and EPO, posphorylation of STAT3 by hyperglycemia needs a longer time in incubation. It seems that enough oxidative stress induced by hyperglycemia needs a time to accumulate and STAT3 phosphorylation via oxidative stress is varied with that rapidly induced via the p-JAK2. Taken together, we found that expression of STAT3 increased by hyperglycemia is mainly through oxidative stress to promote the expressions of fibrosis-related signals, including CTGF and MMP-9, in H9c2 cells. This mechanism is quite different with that induced by receptor activation, both LPS and EPO.

In conclusion, we identified that STAT3 phosphorylation is rapidly raised by LPS or EPO via receptor-mediated signaling, but different from high glucose, in H9c2 cells. Additionally, STAT3 increased by hyperglycemia needs a longer time because it is mainly through an accumulation of 

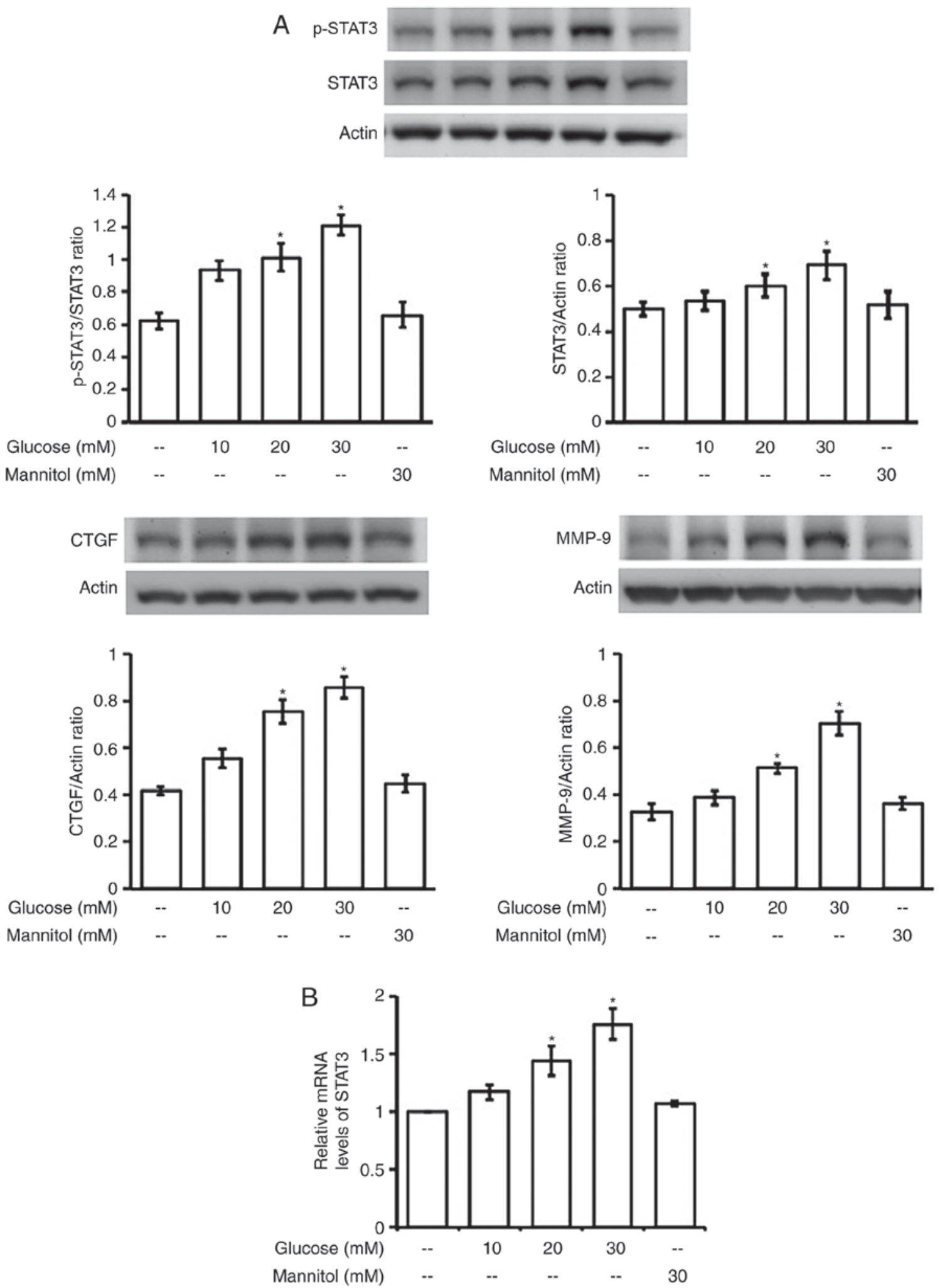

Figure 4. Effects of high glucose on changes in expressions of STAT3 and associated downstream signals in H9c2 cells. (A) The representative changes in p-STAT3, STAT3, CTGF and MMP-9 expressions after a 24-h incubation with high glucose at the indicated concentration are shown in the upper panel. Similar incubation with manitol $(30 \mathrm{mM})$ was demonstrated to produce the same osmolarity as high glucose $(30 \mathrm{mM})$. The protein levels, using p-STAT3 over STAT3 or each signal over $\beta$-actin, are indicated as mean \pm SEM ( $n=4$ per group) in each column. (B) Related mRNA expression as detected using RT-PCR and the quantified mRNA level of STAT3 is represented as mean \pm SEM ( $n=6$ per group). " $\mathrm{P}<0.05$ compared to the control incubated in normal medium (at 0 concentration of glucose). STAT3, signal transducer and activator of transcription 3; p-, phosphorylated; CTGF, connective tissue growth factor; MMP-9, matrix metalloproteinase-9; SEM, standard error of the mean.

oxidative stress which is effective to promote the transcription of downstream signals for fibrosis, including CTGF and
MMP-9, in H9c2 cells. Therefore, we suggest that phosphorylation of STAT3 seems suitable for rapidly identification of 


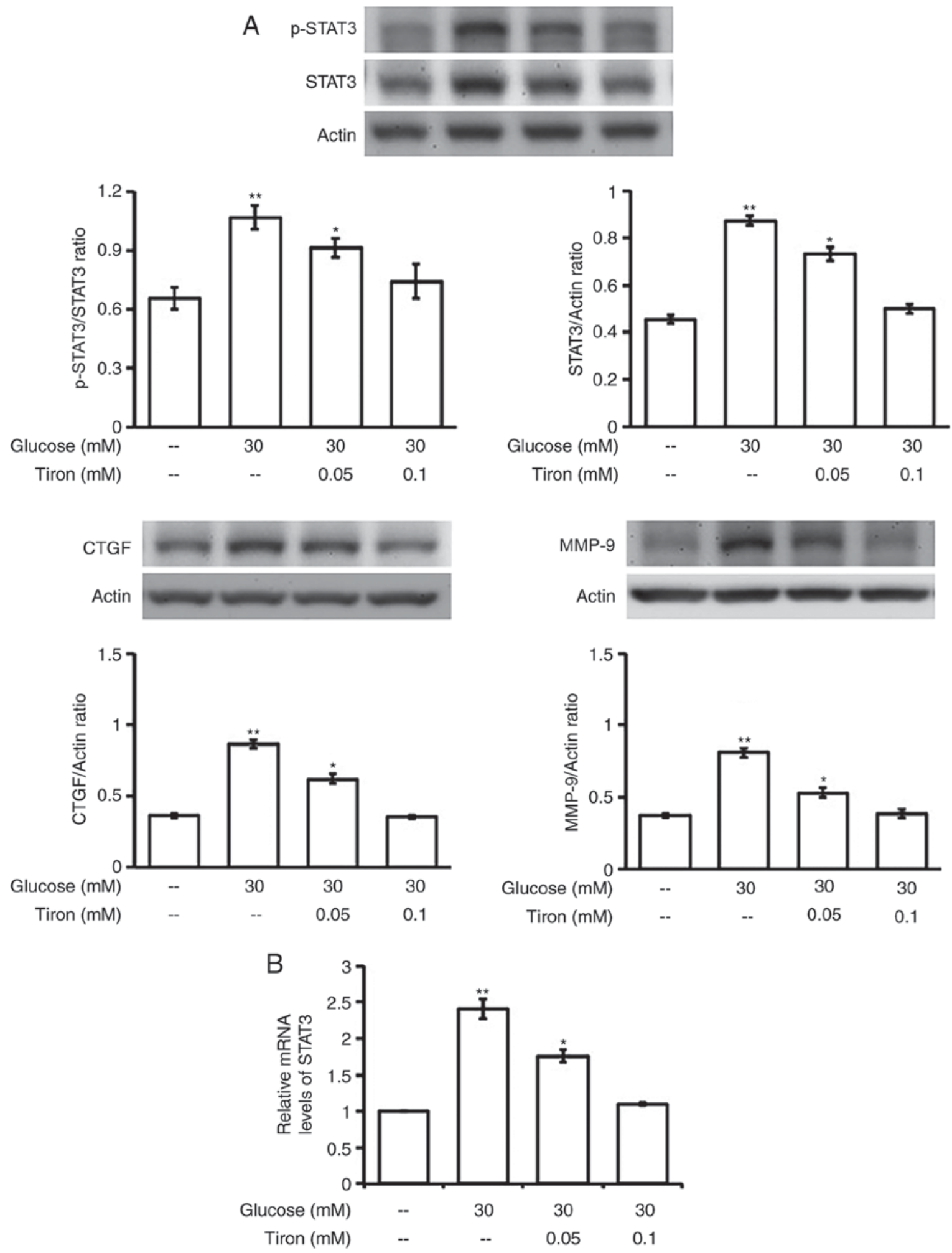

Figure 5. Effects of antioxidant tiron on the hyperglycemia-induced changes in expressions of STAT3 and associated downstream signals in H9c2 cells. (A) The representative changes in signal expressions modified by tiron at indicated two concentrations after a 24-h incubation with high glucose (30 mM) shown in the upper panel and the protein levels are indicated as mean \pm SEM ( $\mathrm{n}=4$ per group) in each column. (B) Related expression of the quantified mRNA level for STAT3 by RT-PCR is represented as mean \pm SEM ( $\mathrm{n}=6$ per group). ${ }^{*} \mathrm{P}<0.05$ and ${ }^{* *} \mathrm{P}<0.01$ compared to the control that incubated in normal medium (first column), respectively. STAT3, signal transducer and activator of transcription 3; p-, phosphorylated; CTGF, connective tissue growth factor; MMP-9, matrix metalloproteinase-9; SEM, standard error of the mean.

receptor-mediated signaling while the nuclear STAT3 is more reliable in cardiac cells receiving hyperglycemic stress. Furthermore, nuclear STAT3 may be a potential clinical indicator of cardiac fibrosis and heart dysfunction. The developments of new drugs that not only prevent myofibroblast formation but also alleviate the hyperglycemia-induced STAT3 phosphorylation may be useful to prevent cardiac dysfunction. 


\section{Acknowledgements}

We thank Y.C. Chen for assistance with the experiments, and we acknowledge Jake Carpenter for editing. The present study was partially supported by a grant from the Chi-Mei Medical Center-Liouying (CLFHR10407), Tainan, Taiwan, R.O.C.

\section{References}

1. Haghikia A, Ricke-Hoch M, Stapel B, Gorst I and HilfikerKleiner D: STAT3, a key regulator of cell-to-cell communication in the heart. Cardiovasc Res 102: 281-289, 2014.

2. Boengler K, Hilfiker-Kleiner D, Heusch G and Schulz R: Inhibition of permeability transition pore opening by mitochondrial STAT3 and its role in myocardial ischemia/reperfusion. Basic Res Cardiol 105: 771-785, 2010.

3. Elschami M and Scherr M, Philippens B and Gerardy-Schahn R: Reduction of STAT3 expression induces mitochondrial dysfunction and autophagy in cardiac HL-1 cells. Eur J Cell Biol 92: 21-29, 2013.

4. Heusch G, Musiolik J, Gedik N and Skyschally A: Mitochondrial STAT3 activation and cardioprotection by ischemic postconditioning in pigs with regional myocardial ischemia/reperfusion. Circ Res 109: 1302-1308, 2011

5. Wegrzyn J, Potla R, Chwae YJ, Sepuri NB, Zhang Q, Koeck T, Derecka M, Szczepanek K, Szelag M, Gornicka A, et al: Function of mitochondrial Stat3 in cellular respiration. Science 323 793-797, 2009.

6. Haghikia A, Stapel B, Hoch M and Hilfiker-Kleiner D: STAT3 and cardiac remodeling. Heart Fail Rev 16: 35-47, 2011.

7. Hilfiker-Kleiner D, Hilfiker A, Fuchs M, Kaminski K, Schaefer A, Schieffer B, Hillmer A, Schmiedl A, Ding Z, Podewski E, et al: Signal transducer and activator of transcription 3 is required for myocardial capillary growth, control of interstitial matrix deposition and heart protection from ischemic injury. Circ Res 95: 187-195, 2004.

8. Zouein FA, Kurdi M and Booz GW: Dancing rhinos in stilettos: The amazing saga of the genomic and nongenomic actions of STAT3 in the heart. JAKSTAT 2: e24352, 2013.

9. Wang S, Li B, Li C, Cui W and Miao L: Potential renoprotective agents through inhibiting CTGF/CCN2 in diabetic nephropathy. J Diabetes Res 2015: 962383, 2015.

10. Miller AM, Wang H, Bertola A, Park O, Horiguchi N, Ki SH, Yin S, Lafdil $\mathrm{F}$ and Gao B: Inflammation-associated interleukin-6/signal transducer and activator of transcription 3 activation ameliorates alcoholic and nonalcoholic fatty liver diseases in interleukin-10-deficient mice. Hepatology 54: 846-856, 2011

11. Jung JE, Lee HG, Cho IH, Chung DH, Yoon SH, Yang YM, Lee JW, Choi S, Park JW, Ye SK and Chung MH: STAT3 is a potential modulator of HIF-1-mediated VEGF expression in human renal carcinoma cells. FASEB J 19: 1296-1298, 2005.

12. Chen Y, Wang JJ, Li J, Hosoya KI, Ratan R, Townes T and Zhang SX: Activating transcription factor 4 mediates hyperglycaemia-induced endothelial inflammation and retinal vascular leakage through activation of STAT3 in a mouse model of type 1 diabetes. Diabetologia 55: 2533-2545, 2012.

13. Saengboonmee C, Seubwai W, Pairojkul C and Wongkham S: High glucose enhances progression of cholangiocarcinoma cells via STAT3 activation. Sci Rep 6: 18995, 2016.

14. Liu L, McBride KM and Reich NC: STAT3 nuclear import is independent of tyrosine phosphorylation and mediated by importin-alpha3. Proc Natl Acad Sci USA 102: 8150-8155, 2005

15. Yang J, Chatterjee-Kishore M, Staugaitis SM, Nguyen H, Schlessinger K, Levy DE and Stark GR: Novel roles of unphosphorylated STAT3 in oncogenesis and transcriptional regulation. Cancer Res 65: 939-947, 2005.

16. Meldrum DR: Tumor necrosis factor in the heart. Am J Physiol 274: R577-R595, 1998

17. Wagner DR, McTiernan C, Sanders VJ and Feldman AM: Adenosine inhibits lipopolysaccharide-induced secretion of tumor necrosis factor-alpha in the failing human heart. Circulation 97: 521-524, 1998.

18. Cowan DB, Poutias DN, Del Nido PJ and McGowan FX Jr: CD14-independent activation of cardiomyocyte signal transduction by bacterial endotoxin. Am J Physiol Heart Circ Physiol 279: H619-H629, 2000
19. Darnell JE Jr: STATs and gene regulation. Science 277: 1630-1635, 1997.

20. Ruff-Jamison S, Zhong Z, Wen Z, Chen K, Darnell JE Jr and Cohen S: Epidermal growth factor and lipopolysaccharide activate Stat 3 transcription factor in mouse liver. J Biol Chem 269: 21933-21935, 1994.

21. Chow JC, Young DW, Golenbock DT, Christ WJ and Gusovsky F: Toll-like receptor-4 mediates lipopolysaccharide-induced signal transduction. J Biol Chem 274: 10689-10692, 1999.

22. Avlas O, Fallach R, Shainberg A, Porat E and Hochhauser E: Toll-like receptor 4 stimulation initiates an inflammatory response that decreases cardiomyocyte contractility. Antioxid Redox Signal 15: 1895-1909, 2011.

23. Seavey MM and Dobrzanski P: The many faces of Janus kinase. Biochem Pharmacol 83: 1136-1145, 2012.

24. Xiao L, Li Z, Xu P, Li Z, Xu J and Yang Z: The expression of EPOR in renal cortex during postnatal development. PloS one 7: e41993, 2012.

25. Yu X, Shacka JJ, Eells JB, Suarez-Quian C, Przygodzki RM, Beleslin-Cokic B, Lin CS, Nikodem VM, Hempstead B, Flanders KC, et al: Erythropoietin receptor signalling is required for normal brain development. Development 129: 505-516, 2002.

26. Qiao S, Mao X, Wang Y, Lei S, Liu Y, Wang T, Wong GT, Cheung CW, Xia Z, Irwin MG, et al: Remifentanil preconditioning reduces postischemic myocardial infarction and improves left ventricular performance via activation of the janus activated kinase-2/signal transducers and activators of transcription-3 signal pathway and subsequent inhibition of glycogen synthase kinase-3beta in rats. Crit Care Med 44: e131-e145, 2016.

27. Fenton MJ and Golenbock DT: LPS-binding proteins and receptors. J Leukoc Biol 64: 25-32, 1998.

28. Dai B, Cui M, Zhu M, Su WL, Qiu MC and Zhang H: STAT1/3 and ERK1/2 synergistically regulate cardiac fibrosis induced by high glucose. J Leukoc Biol 32: 960-971, 2013.

29. Fiaschi T, Magherini F, Gamberi T, Lucchese G, Faggian G, Modesti A and Modesti PA: Hyperglycemia and angiotensin II cooperate to enhance collagen I deposition by cardiac fibroblasts through a ROS-STAT3-dependent mechanism. Biochim Biophys Acta 1843: 2603-2610, 2014.

30. Wu L, Tan JL, Wang ZH, Chen YX, Gao L, Liu JL, Shi YH, Endoh $\mathrm{M}$ and Yang HT: ROS generated during early reperfusion contribute to intermittent hypobaric hypoxia-afforded cardioprotection against postischemia-induced $\mathrm{Ca}(2+)$ overload and contractile dysfunction via the JAK2/STAT3 pathway. J Mol Cell Cardiol 81: 150-161, 2015

31. Watkins SJ, Borthwick GM and Arthur HM: The H9C2 cell line and primary neonatal cardiomyocyte cells show similar hypertrophic responses in vitro. In Vitro Cell Dev Biol Anim 47: 125-131, 2011.

32. Fan MJ, Huang-Liu R, Shen CY, Ju DT, Lin YM, Pai P, Huang PY, Ho TJ, Tsai FJ, Tsai CH and Huang CY: Reduction of TLR4 mRNA stability and protein expressions through inhibiting cytoplasmic translocation of HuR transcription factor by $\mathrm{E}_{2}$ and/or ER $\alpha$ in LPS-treated H9c2 cardiomyoblast cells. Chin J Physiol 57: 8-18, 2014.

33. Parvin A, Pranap R, Shalini U, Devendran A, Baker JE and Dhanasekaran A: Erythropoietin protects cardiomyocytes from cell death during hypoxia/reperfusion injury through activation of survival signaling pathways. PLoS One 9: e107453, 2014.

34. Yang L, Luo C, Chen C, Wang X, Shi W and Liu J: All-trans retinoic acid protects against doxorubicin-induced cardiotoxicity by activating the ERK2 signalling pathway. Br J Pharmacol 173: 357-371, 2016.

35. Mar GY, Ku PM, Chen LJ, Cheng KC, Li YX and Cheng JT: Increase in cardiac M2-muscarinic receptor expression is regulated by GATA binding protein 4 (GATA-4) in streptozotocin-induced diabetic rats. Int J Cardiol 167: 436-441, 2013.

36. Cheng YZ, Chen LJ, Lee WJ, Chen MF, Jung Lin H and Cheng JT: Increase of myocardial performance by rhodiola-ethanol extract in diabetic rats. J Ethnopharmacol 144: 234-239, 2012.

37. Cheng JT, Yu BC and Tong YC: Changes of M3-muscarinic receptor protein and mRNA expressions in the bladder urothelium and muscle layer of streptozotocin-induced diabetic rats. Neurosci Lett 423: 1-5, 2007.

38. Carraway MS, Suliman HB, Jones WS, Chen CW, Babiker A and Piantadosi CA: Erythropoietin activates mitochondrial biogenesis and couples red cell mass to mitochondrial mass in the heart. Circ Res 106: 1722-1730, 2010. 
39. Mudalagiri NR, Mocanu MM, Di Salvo C, Kolvekar S, Hayward M, Yap J, Keogh B and Yellon DM: Erythropoietin protects the human myocardium against hypoxia/reoxygenation injury via phosphatidylinositol-3 kinase and ERK1/2 activation. Br J Pharmacol 153: 50-56, 2008.

40. Cimolai MC, Alvarez S, Bode C and Bugger H: Mitochondrial mechanisms in septic cardiomyopathy. Int J Mol Sci 16: 17763-17778, 2015.

41. Desco MC, Asensi M, Márquez R, Martínez-Valls J, Vento M, Pallardó FV, Sastre J and Viña J: Xanthine oxidase is involved in free radical production in type 1 diabetes: Protection by allopurinol. Diabetes 51: 1118-1124, 2002.

42. MacKichan ML and DeFranco AL: Role of ceramide in lipopolysaccharide (LPS)-induced signaling. LPS increases ceramide rather than acting as a structural homolog. J Biol Chem 274 $1767-1775,1999$

43. Meng X, Ao L, Brown JM, Meldrum DR, Sheridan BC, Cain BS, Banerjee A and Harken AH: LPS induces late cardiac functional protection against ischemia independent of cardiac and circulating TNF-alpha. Am J Physiol 273: H1894-H1902, 1997.

44. Li L, Takemura G,Li Y, Miyata S, Esaki M,Okada H, Kanamori H, Khai NC, Maruyama R, Ogino A, et al: Preventive effect of erythropoietin on cardiac dysfunction in doxorubicin-induced cardiomyopathy. Circulation 113: 535-543, 2006

45. Lipsic E, Westenbrink BD, van der Meer P, van der Harst $P$ Voors AA, van Veldhuisen DJ, Schoemaker RG and van Gilst WH: Low-dose erythropoietin improves cardiac function in experimental heart failure without increasing haematocrit. Eur J Heart Fail 10: 22-29, 2008.

46. Shravah J, Wang B, Pavlovic M, Kumar U, Chen DD, Luo H and Ansley DM: Propofol mediates signal transducer and activator of transcription 3 activation and crosstalk with phosphoinositide 3-kinase/AKT. JAKSTAT 3: e29554, 2014.

47. Gross ER, Hsu AK and Gross GJ: The JAK/STAT pathway is essential for opioid-induced cardioprotection: JAK2 as a mediator of STAT3, Akt and GSK-3 beta. Am J Physiol Heart Circ Physiol 291: H827-H834, 2006.

48. Yu H, Lee H, Herrmann A, Buettner R and Jove R: Revisiting STAT3 signalling in cancer: New and unexpected biological functions. Nat Rev Cancer 14: 736-746, 2014.

49. Gu JJ, Montealegre ZF, Robert D, Engel MS, Qiao GX and Ren D Wing stridulation in a Jurassic katydid (Insecta, Orthoptera) produced low-pitched musical calls to attract females. Proc Nat Acad Sci USA 109: 3868-3873, 2012.
50. Asbun J, Manso AM and Villarreal FJ: Profibrotic influence of high glucose concentration on cardiac fibroblast functions: Effects of losartan and vitamin E. Am J Physiol Heart Circ Physiol 288: H227-H234, 2005.

51. Suskin N, McKelvie RS, Burns RJ, Latini R, Pericak D, Probstfield J, Rouleau JL, Sigouin C, Solymoss CB, Tsuyuki R, et al: Glucose and insulin abnormalities relate to functional capacity in patients with congestive heart failure. Eur Heart J 21: $1368-1375,2000$

52. Ather S, Chan W, Bozkurt B, Aguilar D, Ramasubbu K, Zachariah AA, Wehrens XH and Deswal A: Impact of noncardiac comorbidities on morbidity and mortality in a predominantly male population with heart failure and preserved versus reduced ejection fraction. J Am Coll Cardiol 59: 998-1005, 2012.

53. Paulus WJ and Tschöpe C: A novel paradigm for heart failure with preserved ejection fraction: Comorbidities drive myocardial dysfunction and remodeling through coronary microvascular endothelial inflammation. J Am Coll Cardiol 62: 263-271, 2013.

54. Ma H, Gong H, Chen Z, Liang Y, Yuan J, Zhang G, Wu J, Ye Y, Yang C, Nakai A, et al: Association of Stat3 with HSF1 plays a critical role in G-CSF-induced cardio-protection against ischemia/reperfusion injury. J Mol Cell Cardiol 52: 1282-1290, 2012.

55. Butler KL, Huffman LC, Koch SE, Hahn HS and Gwathmey JK: STAT-3 activation is necessary for ischemic preconditioning in hypertrophied myocardium. Am J Physiol Heart Circ Physiol 291: H797-H803, 2006.

56. Roul D and Recchia FA: Metabolic alterations induce oxidative stress in diabetic and failing hearts: Different pathways, same outcome. Antioxid Redox Signal 22: 1502-1514, 2015.

57. Varga ZV, Giricz Z, Liaudet L, Hasko G, Ferdinandy P and Pacher P: Interplay of oxidative, nitrosative/nitrative stress, inflammation, cell death and autophagy in diabetic cardiomyopathy. Biochim Biophys Acta 1852: 232-242, 2015.

58. Monticone M, Taherian R, Stigliani S, Carra E, Monteghirfo S, Longo L, Daga A, Dono M, Zupo S, Giaretti W and Castagnola P: NAC, tiron and trolox impair survival of cell cultures containing glioblastoma tumorigenic initiating cells by inhibition of cell cycle progression. PLoS One 9: e90085, 2014. 